\title{
Interférences
}

Ars scribendi

$6 \mid 2012$

La question de la littérarité

\section{La reconnaissance du poétique dans le De poematibus de Diomède}

\section{Marcos Martinho}

\section{(2) OpenEdition}

1 Journals

\section{Édition électronique}

URL : http://journals.openedition.org/interferences/203

DOI : 10.4000/interferences.203

ISSN : $1777-5485$

Éditeur

HiSoMA - Histoire et sources des Mondes antiques

\section{Édition imprimée}

Date de publication : 1 janvier 2012

ISSN : 1777-5485

\section{Référence électronique}

Marcos Martinho, «La reconnaissance du poétique dans le De poematibus de Diomède ", Interférences [En ligne], 6| 2012, mis en ligne le 10 juillet 2014, consulté le 15 septembre 2020. URL : http:// journals.openedition.org/interferences/203; DOI : https://doi.org/10.4000/interferences.203

Ce document a été généré automatiquement le 15 septembre 2020.

Tous droits réservés 


\title{
La reconnaissance du poétique dans le De poematibus de Diomède
}

\author{
Marcos Martinho
}

\section{Introduction}

1 Dans le Livre III de l'Ars grammatica de Diomède, on trouve une section intitulée De poematibus (GL I 482, 13 -492, 14). Le grammairien y expose d'abord deux façons de distinguer les poèmes : l'une selon les genera et species (GL I 482, $14-483,6$ ), et l'autre selon les kharaktêres (GL I 483, 7-26); puis il expose les carmina : epos (GL I 483, 27 - 484, 17), elegia (GL I 484, 17 - 485, 10), iambus (GL I 485, 11-29), satira (GL I 485, 30 - 486, 16), bucolica (GL I 486, 17 - 487, 10), tragoedia (GL I 487, 11 - 488, 2), comoedia (GL I 488, 3 - 491, $2 ; 491,20$ - 492, 14), satyrica (GL I 491, 3-12), mimus (GL I 491, 13-19). L'exposé des genera et species de poèmes, en particulier, provient de sources grecques, les unes platoniciennes, les autres aristotéliciennes, où l'on peut découvrir une dispute sur la spécificité du poétique. Dans ce qui suit, je me propose de préciser quelles sont ces sources et quelle est cette dispute, afin de montrer que la leçon de Diomède sur les genera et species de poèmes renferme un débat sur la reconnaissance du poétique.

\section{Les sources : la République de Platon et la Poétique d'Aristote}

Dans l'exposé des genera et species de poèmes, Diomède distingue trois genera, dont il donne le nom, une définition et des exemples.

$1^{\mathrm{er}}$ genus

- nom : lat. actiuum ou imitatiuum = gr. dramatikón ou mimetikón

- définition : où il n'y a que les personnages qui agissent, sans l'intervention du poète

- exemples : tragédies et comédies + Verg., B. I ; IX

$2^{\mathrm{e}}$ genus

- nom : lat. enarratiuum ou enuntiatiuum = gr. exegetikón ou apaggeltikón 
- définition : où il n'y a que le poète qui parle, sans l'intervention des personnages

- exemples : Verg., G. I-III ; IV 1-316 ; Lucr.

$3^{\mathrm{e}}$ genus

- nom : lat. commune ou mixtum = gr. koinón ou miktón

- définition : où le poète parle, et des personnages sont introduits en train de parler

- exemples : Hom., Il. ; Od. ; Verg., Aen.

Diomède renvoie chaque nom latin à son équivalent grec ${ }^{1}$, de sorte qu'on peut déduire qu'il provient d'une source grecque. Or, dans le Livre III de la République de Platon, Socrate postule que tout ce qui est dit par les conteurs de fables et les poètes est un récit, qu'ils réalisent soit par un récit simple, soit par un récit imitatif, soit en associant les deux (Plat., $R$. III 392 d). Puis il illustre chaque façon de raconter en disant que, dans la poésie et les fables, il y en a une qui s'accomplit entièrement par l'imitation, et qui se rencontre dans la tragédie et la comédie; une autre qui s'accomplit par l'énoncé du poète lui-même, et qui se rencontre dans le dithyrambe ; une troisième qui s'accomplit par les deux modes, et qui se rencontre dans l'épopée mais aussi ailleurs (Plat., $R$. III 394 b-c; cf. 396 e-397 b). Si l'on compare la leçon de Diomède avec celle de la République, on remarque que :

a. quant à la définition :

Socrate et Diomède distinguent trois genres de poésie : un où il n'y a que le poète qui parle; un autre où il n'y a que les personnages qui parlent; un troisième où parfois c'est l'un, parfois ce sont les autres qui interviennent ;

b. quant au nom :

Socrate dit que dans l'apaggelía du poète il n'y a que diégesis, sans mímesis, et que dans les rhéseis des personnages il y a diégesis par le moyen de la mímesis. Diomède, à son tour, appelle un genre appageltikós ou exegetikós, et un autre, mimetikós ;

c. quant aux exemples :

Socrate et Diomède illustrent le genre mimétique ou imitatif par la tragédie et la comédie, et le genre mixte ou commun, par l'épopée et, en particulier, l'Iliade.

On peut donc conclure que, de façon directe ou indirecte, la leçon de Diomède vient de celle de la République de Platon.

Outre cette filiation, on peut en déceler également une autre dans l'exposé des kharaktêres et des carmina, lequel, comme il a été dit, fait suite à celui des genera et species de poèmes. Or, quand il expose les neuf carmina, Diomède en définit quatre en grec, à savoir : épopée ${ }^{2}$, tragédie ${ }^{3}$, comédie ${ }^{4}$, mime ${ }^{5}$. Trois fois il renvoie la définition, de façon anonyme, aux Grecs $^{6}$, une fois pourtant il l'attribue nominalement à Théophraste ${ }^{7}$. Mais, justement quand il présente les genera de poèmes - actiuum ou imitatiuum, enarratiuum ou enuntiatiuum, commune ou mixtum -, Diomède ne fait aucune mention de Théophraste, ni plus tard, quand il énumère les kharaktêres de poèmes makrós, brakhýs, mésos, antherós. Pourtant, pour ce qui est des trois premiers caractères - makrós, brakhýs et mésos-, Ammonius évoque Théophraste, pour dire que celui-ci a distingué deux rapports du discours - l'un avec l'auditeur, propre à la poétique et à la rhétorique, et l'autre avec l'affaire, propre au philosophe - et qu'il a énoncé alors que, si le philosophe s'applique à démontrer ce qui est vrai et réfuter ce qui est faux, la tâche de la poétique et de la rhétorique est quant à elle de choisir les mots les plus nobles et de les lier les uns aux autres de façon harmonieuse, afin qu'ils plaisent à l'auditeur, qu'ils le surprennent et le contraignent à la persuasion grâce à la clarté, la douceur et 
les autres espèces de discours et aussi, justement, grâce à la makrología et la brakhylogía 8 .

Quant au caractère antherós qui, à la vérité, appartient à l'exposé de trois autres caractères - à savoir hadrós, iskhnós et mésos -, Denys d'Halicarnasse mentionne Théophraste justement au moment où il s'attache à les distinguer (cf. D.H., Dem. 1, 1;2, $8 ; 8,1$ ). En effet, après avoir décrit deux kharaktêres contraires (cf. D.H., Dem. 2, 8) celui de Thucydide, insolite, recherché, fortement structuré, plein de toutes sortes d'ornements ajoutés (D.H., Dem.1, 3), et celui de Lysias, uni, simple, qui doit apparemment sa mise en œuvre et sa vigueur à sa ressemblance avec le parler ordinaire (D.H., Dem. 2, 1) -, Denys passe au kharaktér mixte et composé des deux premiers (D.H., Dem. 3,1$)$, et fait alors mention de Théophraste qui est, selon Thrasimaque, le premier à l'avoir harmonisé et établi dans son éclat actuel (D.H., Dem. 3, 1; cf. 14, 1; Th., frg. Fortenbaugh 685). Du reste, Denys d'Halicarnasse emploie l'adjectif antherós pour qualifier les genres de discours; ou plutôt, il dit de l'harmonie austère qu'elle est la moins "fleurie ${ }^{9}$ ", et d'Isocrate, un des inventeurs du kharaktér mixte aux côtés de Thrasymaque (cf. D.H., Dem. 14, 1), qu'il manque parfois de convenance parce qu'il veut à tout prix que son langage soit « fleuri ${ }^{10}$ ».

Quant aux genera de poèmes enfin, ni Diomède ni aucun autre auteur ne témoignent de ce que Théophraste en ait donné les règles; la distinction des modes de discours était pourtant certainement connue de Théophraste puisque, comme on sait, elle a été exposée par son maître. En effet, dans l'exorde de la Poétique, Aristote énumère l'épopée, la tragédie et la comédie, le dithyrambe, la plus grande partie de l'art de la flûte et de la cithare, pour dire qu'ils ont ceci de commun qu'ils sont des imitations, mais qu'ils diffèrent les uns des autres par trois éléments, à savoir : parce qu'on imite en des choses différentes (en hetérois), parce qu'on imite des choses différentes (hétera), parce qu'on imite de façon différente (hetéros) (Arist., Po. 1, 1447 a 13-18); en d'autres termes, par le moyen, l'objet et le mode d'imitation. Puis, dans les trois premiers chapitres, Aristote examine les trois différences ${ }^{11}$ grâce auxquelles il distingue à la fois les imitations les unes des autres, et en particulier l'imitation poétique, et les espèces poétiques les unes des autres, c'est-à-dire l'épopée et la tragédie, les iambes et la comédie. Le mode d'imitation, à son tour, est double, car ou bien quelqu'un imite en racontant - qu'il devienne un autre ou qu'il reste lui-même sans changement -, ou bien quelques-uns imitent en agissant et en opérant quelque chose ${ }^{12}$. Après avoir exposé ces différences, Aristote donne un bref résumé de l'origine et du développement de la tragédie et de la comédie (Arist., Po. 4-5), puis il expose plus longuement la tragédie (Arist., Po. 6-22), et plus brièvement l'épopée (Arist., Po. 23-24), et la Poétique s'achève sur une comparaison entre l'une et l'autre (Arist., Po. 26).

7 Cela dit, l'exposé des poèmes de Diomède semble s'accorder avec la Poétique d'Aristote, dans la mesure où tous deux établissent d'abord les façons de discerner les espèces poétiques avant de les présenter :

a. quant à la façon de discerner les espèces poétiques :

Aristote expose trois différences : le moyen, l'objet et le mode de l'imitation poétique, et divise le mode en deux : l'énoncé du poète, qui raconte ce que les personnages ont fait ou rapporte ce qu'ils ont dit, et l'action et opération des acteurs, qui agissent et parlent comme des personnages. Diomède, à son tour, expose deux façons de classer les poèmes : selon les genera et species, et selon les kharaktêres, et divise les genera en trois : celui où il n'y a que le poète qui parle, celui où il n'y a que les personnages qui agissent, 
celui où parfois le poète parle et où parfois les personnages sont introduits en train de parler ;

b. quant à l'exposé des espèces poétiques :

Aristote se borne aux espèces de la tragédie et de l'épopée, de l'iambe et de la comédie, tandis que Diomède énumère neuf carmina. Cependant, tous deux dirigent leur attention vers l'histoire de la naissance et du développement de la tragédie et de la comédie, ainsi que vers l'explication étymologique de komoidía et drâma :

b.1. quant à la naissance et au développement de la tragédie et de la comédie :

Aristote raconte qu'Eschyle a porté d'un à deux le nombre des acteurs, a réduit la partie du chœur et a donné la première place au dialogue, tandis que Sophocle a introduit trois acteurs ainsi que le décor de la scène (Arist., Po. 4, 1449 a 15-19); de la comédie, pourtant, Aristote dit qu'on ignore qui lui a donné les masques, le prologue, le nombre des acteurs, etc., mais qu'on attribue à Épicharme son invention (Arist., Po. 5, 1449 a 37 b 9). Diomède, à son tour, remarque que, si dans le drame grec, en général, les personnages ne dépassent pas le nombre de trois, les auteurs latins ont toutefois introduit des personnages plus nombreux (GL I 490, 27 - 491, 3 ; cf. 491, 22-29). En outre, Diomède raconte que, dans un premier temps, tous jouaient un rôle dans la comédie, car aussi bien le pantomimus que le pythaules et le choraules y chantaient; mais, parce qu'il n'était pas possible que tous se distinguassent dans tous les rôles, les acteurs commencèrent à revendiquer la première place; ainsi, pour éviter de céder, les pantomimi quittèrent les autres personnages et finirent par renoncer à la comédie (GL I 491, $30-492,9)$. Enfin, Diomède fait allusion à d'autres auteurs qui considèrent qu'Épicharme fut le premier à composer des comédies (GL I 489, 8-10);

b.2. quant à l'explication étymologique de komoidía et drâma :

Aristote attribue aux Doriens l'explication selon laquelle komoidós dérive de komázein, qui vient de kóme - parce qu'il leur était interdit de séjourner dans les villages, les participants allaient de kóme en kóme, "de village en village", de sorte qu'on les a appelés komoidoí -, et drâma dérive de drân (Arist., Po. 3, 1448 a 29 - b 2). Diomède, à son tour, rapporte d'après Varron quatre explications étymologiques de komoidía: 1. komoidía vient de kóme - parce que les jeunes Athéniens avaient coutume d'aller aux alentours des kómai, "villages ", et d'y exécuter un type de chant que l'on a appelé komoidía ; 2. komoidía vient de kóme kaì oidé, «village et chant»-parce que, ayant quitté la campagne pour venir s'installer à Athènes, les jeux des kómai, "villages", commencèrent à être chantés, de sorte qu'on les a appelés kóme kaì oidé; 3. komoidía vient de kóme - parce que la komoidía, « comédie », décrit le sort des kómai, «villages », c'est-à-dire des maisons humbles, et non, comme la tragédie, celui des maisons publiques et royales; 4. komoidía vient de kômos - parce que les kômoi, «festins " des jeunes amoureux, sont fréquents dans le genre de pièce qui s'est appelé komoidía (GL I 488, 5-14; cf. Varr., De poem. frg. Funaioli 305). Quant à drâma, Diomède dit tout simplement qu'il vient de drân (GL I 490, 21).

8 En résumé, la leçon de Diomède sur les poèmes semble provenir, de façon directe ou indirecte, de la République de Platon et de la Poétique d'Aristote: de celle-là, par le nombre, la définition, les noms et les exemples des genres du discours; de celle-ci, par la division binaire de l'exposé, dont la première partie décrit les façons de discerner les espèces poétiques que la deuxième expose. Si l'on compare pourtant la leçon de la Poétique avec celle de la République, on peut déceler une polémique d'Aristote avec Platon, où celui-là dispute avec celui-ci sur la spécificité du poétique. Cette polémique 
permet de découvrir les difficultés qui pèsent, en particulier, sur la leçon de Diomède sur les genres de poèmes.

\section{La dispute sur la reconnaissance du poétique}

\section{La dispute aristotélicienne}

Dans le Livre III de la République, d'une part, Socrate distingue deux parties dans l'Iliade : l'apaggelía, « énoncé » du poète, et les rhéseis, "paroles » des personnages (Plat., $R$. III 393 b-c, 394 b). Il dit qu'il y a récit à la fois dans l'énoncé, quand Homère raconte ce que les personnages ont fait, et dans les paroles, quand il rapporte ce qu'ils ont dit (Plat., R. III 393 b) ; dans le second cas, pourtant, il y a récit par le moyen de l'imitation, et dans le premier, récit simple, c'est-à-dire sans imitation (Plat., R. III 393 c-d, 394 a). En d'autres termes, il y a récit par le moyen de l'imitation quand le poète parle comme un autre que lui-même, c'est-à-dire quand il raconte quelque chose d'après l'énoncé d'un personnage, et il y a récit simple, sans imitation, quand le poète parle comme luimême, c'est-à-dire quand il raconte quelque chose d'après son propre énoncé (Plat., R. III $393 \mathrm{a}-\mathrm{b}$; cf. III 394c : di' apaggelías autoû toû poietoû). Dans les trois premiers chapitres de la Poétique, d'autre part, Aristote examine trois différences, grâce auxquelles il distingue, en général, les imitations les unes des autres, et sépare, en particulier, la poétique des autres imitations, et les espèces poétiques les unes des autres : épopée et tragédie, iambes et comédie. Les modes d'imitation, en particulier, servent à distinguer l'une de l'autre les espèces poétiques qui imitent des agents supérieurs, à savoir : l'épopée, imitation narrative, où le rhapsode, unique, imite en énonçant ce qu'ont dit et ce qu'ont fait les personnages, et la tragédie, imitation dramatique, où les acteurs, jusqu'à trois, imitent en agissant et en opérant comme des personnages ${ }^{13}$. En bref, telle est la différence entre la leçon de la République et celle de la Poétique: là Socrate distingue deux façons de raconter, ici Aristote distingue deux façons d'imiter; de sorte que pour Socrate il y a toujours récit, lequel peut être imitatif ou non imitatif, et pour Aristote il y a toujours imitation, laquelle peut être narrative ou dramatique.

Aristote insiste sur la spécificité imitative de la poésie et du poète. Dans le chapitre 1, où il distingue la poésie de la science naturelle (Arist., Po. 1, 1447 a $28-1447 \mathrm{~b} 24$ ), et dans les chapitres 9 et 23, où il distingue la poésie de l'histoire (Arist., Po. 9, 1451 a 36 $1451 \mathrm{~b} 11 ; 23,1459$ a 17-29), Aristote, de manière polémique, contredit les " hommes ${ }^{14}$ » qui appellent poètes sans distinction tous ceux qui composent en vers, et appellent élégiaques ou épiques de façon distincte ceux qui composent en vers élégiaques ou épiques, mais dénient le nom de poète à ceux qui composent en prose. Car, alors, ils les appellent poètes ou non-poètes d'après le vers, et non d'après l'imitation. Pour Aristote, pourtant, on doit appeler poètes tous ceux qui font usage du seul lógos, « discours", pour imiter, que ce soit en prose ou en vers, et, dans ce cas, que ce soit dans un seul mètre, ou dans un mélange de mètres; en revanche, on doit dénier le nom de poète à tous ceux qui font usage du discours pour faire autre chose qu'imiter, que ce soit en prose ou en vers. De cette façon, on doit appeler poète Homère, qui emploie un seul mètre, et Chérémon, qui mélange toutes sortes de mètres, non parce qu'ils font usage du mètre - d'un seul, Homère, ou de plusieurs, Chérémon -, mais parce qu'ils imitent en vers, et pareillement on doit appeler poétiques les mimes de Sophron et de 
Xénarque ainsi que les dialogues socratiques, tout simplement parce qu'ils sont des imitations, en dépit de leur caractère de discours en prose. En revanche, on ne doit appeler poète ni Empédocle, parce qu'il n'imite pas, même s'il fait usage du mètre, ni Hérodote, non pas parce qu'il ne fait pas usage du mètre, mais parce qu'il n'imite pas, car, insiste Aristote, quand l'ouvrage d'Hérodote serait écrit en vers, ce n'en serait pas moins une histoire en vers que ce ne l'est en prose. Autrement dit, Aristote appelle Homère poète, mais Empédocle naturaliste, et Hérodote historien, non d'après le moyen de la composition, qui peut être ou non en vers, mais d'après le dessein de la composition, qui peut être ou non imitative.

11 Il se peut que, parmi d'autres "hommes ", la polémique d'Aristote évoque Platon, ou plutôt, une explication donnée par Socrate dans la République, justement au moment où il distingue récit simple et récit narratif. À la vérité, Socrate inclut dans l'examen des lógoi, « discours» (Plat. R. II 376 e - III 398 b), et ce qui se dit en vers et ce qui se dit en prose ${ }^{15}$, de même qu'Aristote ${ }^{16}$. C'est pourquoi d'ailleurs, quand il illustre récit simple et récit imitatif par Hom., Il. I 22-32 (Plat., R. III 392 d-393 b), il transpose le récit imitatif en vers en un récit simple en prose (Plat., R. III 393 d- 394 b). Pourtant, afin d'expliquer pourquoi, en transposant le récit imitatif en récit simple, il transposera en même temps le mètre en prose, Socrate dit qu'il ne fera pas usage du mètre parce qu'il n'est pas versé en poésie ${ }^{17}$, de sorte qu'il assimile le poétique au métrique. En outre, on remarquera quelques expressions fréquentes dans la leçon de Socrate dans lesquelles les mots poieîn, poíesis et poietés forment un couple avec légein, lógos et logopoiós ${ }^{18}$; car il est possible que ce couple équivaille à celui de mètre et prose, de sorte que Socrate non seulement assimile la poésie au mètre, mais distingue celle-là de la prose.

12 Cela dit, on présume aisément la difficulté de réunir en une seule des leçons sur la spécificité du poétique aussi disparates que celles de Platon et d'Aristote. Avant de revenir à Diomède pour montrer comment cette difficulté pèse sur sa leçon sur les genres et espèces de poèmes, je passe par d'autres auteurs qui parfois distinguent ces leçons avec netteté, parfois les mélangent dans la confusion.

13 C'est avec netteté que Proclus, au moment où il commente le genre de discours d'après lequel la République a été composée (cf. Procl., In R., v. I, p. 14, 1. 15-17), reprend la leçon de Socrate sur les eíde léxeos, "genres d'élocution", en appelant un des genres dramatikòs kaì mimetikós, le deuxième, aphegematikòs kaì amímetos, et le troisième, miktós, de façon à distinguer un genre imitatif et un autre non imitatif (Procl., In R., v. I, p. 14, 1. 18-26). Cette leçon se retrouve dans les scolies de l'Iliade. Afin de commenter le «Catalogue des vaisseaux» (cf. sch. Il. II 494-877, l. 6-7 ; Eust., Il., v. I, p. 399, 1. 14-15), des scolies anonymes et Eusthate (XII ${ }^{\mathrm{e}}$ s. apr. J.-C.) citent pareillement Platon, afin de distinguer trois lógon idéai, « espèces de discours» (sch. Il. II 494-877, 1. 9-12 ; Eust., Il., v. I, p. 400, 1. 16-19), et, d'après ceux-ci, faire remarquer qu'Homère passe de l'espèce mixte à l'espèce non imitative (sch. Il. II 494-877, 1.12-16; Eust., Il., v. I, p. 400, 1. 19 p. 401, 1. 7). Or, ces commentateurs, de même que Proclus, appellent une des espèces mimetikè kaì dramatiké, la deuxième, amímetos, et la troisième, mikté, de façon à distinguer une espèce imitative et une autre non imitative. En bref, c'est parce qu'ils suivent Platon, et non Aristote, que Proclus et les scolies de l'Iliade admettent avec propreté une espèce non imitative de poésie. imitative se retrouve dans un texte tel que le Traité coislinien, qui vient, non de Platon, 
mais d'Aristote, en particulier de la Poétique. En effet, cette filiation s'observe dans la comparaison du plan du Traité coislinien :

$1^{\text {re }}$ partie façons de discerner les espèces de poésie (1-2)

$2^{\mathrm{e}}$ partie comédie (3-18)

1 distinction de la comédie

1.1. distinction entre la comédie, qui évoque le rire, et la tragédie, qui

évoque la peur $(3-4 ;$ cf. 9)

exposé des causes du rire : mots et actions (5) : a. sept façons de produire le rire à partir du mot (5.1-7) ; b. deux ou plusieurs façons de produire le rire à partir des actions (6)

1.2 distinction entre comédie et injure (7-8)

2 division de la comédie

2.1 aspects de la comédie (10-6)

a définition des aspects de la comédie : mûthos (11), êthos (12),

diánoia (13), léxis (14), mélos (15), ópsis (15)

$\mathrm{b}$ occurrence des aspects (16)

2.2 parties de la comédie (17) : prologue (17.1), chœur (17.2),

épisode (17.3), sortie (17.4)

3 espèces de la comédie : ancienne, nouvelle, moyenne (18)

avec le plan de la Poétique d'Aristote :

$1^{\text {re }}$ partie façons de discerner les espèces d'imitation (1-3)

$2^{\mathrm{e}}$ partie imitation poétique

1 origine de l'imitation poétique (4)

2 histoire des espèces poétiques (4)

a développement de la tragédie (4)

b développement de la comédie (5)

$3^{\mathrm{e}}$ partie tragédie et épopée

1 tragédie (6-22)

1.1 définition (6)

1.2 parties qualitatives (cf. 6) : mûthos $(7,16-8)$, éthe (15; cf. 13),

diánoia (cf. 19), léxis (19-22), ópsis (cf. $14 ; 18)$, melopoiía (...)

1.3 parties quantitatives (12)

2 épopée (23-24)

3 problèmes et solutions (25)

4 comparaison entre tragédie et épopée (26)

En dépit d'une telle filiation pourtant, le Traité coislinien divise la poésie en deux catégories, amímetos et mimetiké, "non imitative et imitative»; puis, d'une part, il divise celle-là en historiké et paideutiké, qui, à son tour, se divise en hyphegetiké et theoretiké; d'autre part, il divise la mimetiké en apaggeltikón et dramatikòn kai praktikón, qui, à son tour, se divise en komoidía, tragoidía, mîmoi et sátyroi ${ }^{19}$. Le conflit avec la Poétique est manifeste puisque, bien qu'il en suive le plan général, le Traité coislinien divise la poésie en des parties qui contredisent la conception et la division de la poésie proposées par Aristote. En effet, tandis que celui-ci conçoit la poésie en tant qu'imitation, celui-là admet une poésie non imitative, et, tandis qu'Aristote distingue le poète de l'historien et du naturaliste, le Traité coislinien admet une poésie historique et une autre pédagogique, laquelle, étant données ses divisions - hyphegetiké et theoretiké , fait penser à une poésie philosophique. 
16 Ainsi, après avoir comparé Proclus et les scolies de l'Iliade avec la République, et le Traité coislinien avec la Poétique, je rapproche maintenant Diomède avec les uns et les autres, afin de montrer que sa leçon sur les genres et espèces de poèmes va à l'encontre tantôt de la République, tantôt de la Poétique.

Si, comme il a été dit, les termes et concepts par lesquels Proclus et les scolies de l'Iliade résument la leçon de la République sur les genres d'élocution ou les espèces de discours sont les mêmes, les exemples par lesquels ils les illustrent sont pourtant différents. En effet, tandis que Proclus illustre le genre dramatikòs kaì mimetikós par la comédie et la tragédie, l'aphegematikòs kaì amímetos par le dithyrambe et l'histoire, et le miktós par Homère, les scolies de l'Iliade, toutefois, illustrent l'espèce mimetikè kaì dramatiké par la composition des caractères des personnages, l'amímetos par Phocylide et Théognis, et la mikté par Hésiode. Proclus suit donc de plus près la République, dont il reproduit tous les exemples, en y introduisant celui de l'histoire; les scolies de l'Iliade, quant à elles, introduisent les exemples de Phocylide et Théognis, ainsi que celui d'Hésiode. Ce dernier, en particulier, attire l'attention. Car, tout d'abord, si dans l'examen des discours (Plat., R. II $376 \mathrm{e}$ - III 398 b) Socrate considère à la fois Homère et Hésiode, dont il cite le nom et l'ouvrage (Plat., $R$. II $377 \mathrm{~d}$-e), dans l'examen de l'élocution (Plat., $R$. III $392 \mathrm{c}-398$ b) pourtant, où il distingue justement récit simple, imitatif et mixte, Socrate insiste toujours sur Homère, mais passe sous silence Hésiode. Qui plus est, l'exemple d'Hésiode introduit par Proclus retient l'attention, parce que la Théogonie comme Les Travaux et les Jours seraient plutôt des exemples de discours narratif et non imitatif que de discours mixte. En effet, au moment où il commente Les Travaux et les Jours, c'est par Hésiode que Proclus illustre le caractère narratif, par opposition au caractère dramatique et au caractère mixte, qu'il illustre respectivement par les comédies et la tragédie et par l'Iliade (Procl., Ad Hes. Op., p. 5, l. 8-21). Quoi qu'il en soit, ces exemples se retrouvent chez Diomède, qui illustre une des espèces du genre exegetikós ou apaggeltikós par Théognis, et une autre par Hésiode (GL I 482, $32 ; 483$, 1). Comme ils sont étrangers à la République, on peut conclure que les exemples de Diomède, de Proclus et des scolies remontent à une source intermédiaire entre eux-mêmes et Platon.

Les noms et la division des genres et espèces de poèmes de Diomède, pourtant, peuvent être comparés avec les noms et la division des parties de la poésie du Traité coislinien. Diomède (GL I 482, 26 - 483, 6) distingue :

1. genus actiuum ou imitatiuum = dramatikón ou mimetikón

1.1. species (chez les Grecs) : tragica, comica, satyrica, mimica exemples:...

1.2. species (chez les Romains) : praetexta, tabernaria, Atellana, planipes exemples :...

2. genus enarratiuum ou enuntiatiuum = exegetikón ou apaggeltikón

2.1. species aggeltiké

exemples : sentences (par ex., Theogn.) ; anecdotes

2.2. species historiké

exemples : récits et généalogies (par ex., Hes., Cat. fem.)

2.3. species didaskaliké

exemples : philosophie (par ex., Emped. ; Lucr.) ; astrologie (par ex., Arat. ;

Cic., Phaen.) ; Verg., G.

3. genus commune ou mixtum = koinón ou miktón

3.1. species heroica 
exemples : Hom., Il. ; Verg., Aen.

3.2. species lyrica

exemples : Archil. ; Hor.

et le Traité coislinien (1-2), à son tour, distingue :

1. poíesis amímetos

1.1. historiké

1.2. paideutiké

1.2.1. hyphegetiké

1.2.2. theoretiké

2. poíesis mimetiké

2.1. apaggeltikón

2.2. dramatikòn kaì praktikón

2.2.1. komoidía, tragoidía, mîmoi, sátyroi.

Tout d'abord, ce sont les espèces de la partie dramatique qui attirent l'attention: comédie, tragédie, mimes et drame satyrique, puisque Diomède et le Traité coislinien sont les seuls textes à les énumérer toutes les quatre. En outre, la division de la poésie non imitative en historiké et paideutiké et la division de celle-ci en hyphegetiké et theoretiké, divisions du Traité coislinien, peuvent être comparées avec les trois espèces du genre exegetikós ou apaggeltikós de Diomède ; ainsi, on peut rapprocher l'historiké de l'un et l'historiké de l'autre, l'hyphegetiké et l'apaggeltiké, la paideutiké et la didaskaliké. Comme cependant ils sont étrangers à la Poétique, on peut conclure que les espèces de poèmes de Diomède et les parties de la poésie du Traité coislinien remontent à une source intermédiaire entre eux-mêmes et Aristote.

Cela dit, on présume aisément la difficulté qui pèse sur les espèces que Diomède subordonne aux genres de poème et, pareillement, sur les exemples par lesquels il illustre celles-là. Car du genre exegetikós ou apaggeltikós Diomède distingue trois espèces clairement non imitatives, à savoir : l'aggeltiké, l'historiké et la didaskaliké, et il illustre l' apaggeltiké par Théognis et les anecdotes, l'historiké par les récits et les généalogies, telles que le Catalogue des femmes d'Hésiode, et la didaskaliké par la philosophie, par exemple par Empédocle et Lucrèce, ainsi que par l'astrologie, par exemple par les Phénomènes d'Aratos, ou de Cicéron, et les Géorgiques de Virgile (GL I 482, 31 - 483, 3). De sorte que, en conflit manifeste avec Aristote, Diomède non seulement tient pour poétiques des compositions philosophiques et historiques, mais les tient comme telles justement parce qu'elles sont des compositions en vers ${ }^{20}$; car il faut remarquer que tous les exemples par lesquels il illustre l'espèce historique et l'espèce didascalique, et donc la philosophie, sont des compositions en vers ${ }^{21}$. À la vérité, la leçon sur les genres et espèces de poèmes est une partie du Livre III de l'Ars grammatica, où Diomède expose les leçons de métrique ${ }^{22}$, à savoir: la définition de la poétique (GL I 473, 15-20), la définition du pied (GL I 473, 21-24), la définition du mètre (GL I 474, 1-28), les pieds (GL I 474, 29 - 482, 12), les poèmes (GL I 482, 13 - 492, 14), l'ensemble des terminaisons des noms (GL I 492, 15 - 494, 2), les mètres (GL I 494, 3 - 518, 24) et, en particulier, les mètres d'Horace (GL I 518, 25 - 529, 28); de sorte que Diomède semble considérer l'étude de la poétique comme une partie de celle de la métrique.

21 En résumé, la difficulté qui pèse sur la leçon de Diomède est double puisque, mélangeant des sources d'origine platonicienne et aristotélicienne, elle renferme d'un côté un conflit préexistant, la dispute d'Aristote avec Platon, et établit de l'autre un 
conflit actuel, le désaccord de Diomède lui-même tantôt avec Platon, tantôt avec Aristote.

\section{La dispute post-aristotélicienne}

Comme il a été dit, Diomède semble reprendre une source intermédiaire entre lui et la République, ou entre lui et la Poétique, laquelle source aurait peut-être déjà mélangé l'une à l'autre, en dépit de la dispute d'Aristote avec Platon sur la reconnaissance du poétique. À la vérité, il se peut que Diomède ou sa source dépendent, de façon directe ou indirecte, non de la Poétique, mais du Sur les poètes d'Aristote; car, s'il est vrai que celle-là, écrit acroatikós, s'est circonscrite à l'école péripatéticienne, celui-ci toutefois, dialogue exoterikós, a circulé en dehors de l'école (cf. Gel. XX 5, 1-6). En effet, à en juger par les témoignages et fragments du Sur les poètes, ce dialogue rassemblait quelquesunes des opinions sur la spécificité du poétique qu'on lit dans la Poétique, lesquelles ont circulé parmi les Grecs postérieurs à Aristote, qui ont, à leur tour, prolongé et développé la dispute sur la reconnaissance du poétique. Dans ce qui suit, je me propose d'exposer quelles sont ces sources, et quelle est cette dispute, afin de montrer que, outre le débat aristotélicien sur la reconnaissance du poétique, la leçon de Diomède sur les genera et species de poèmes renferme un autre débat, post-aristotélicien.

\section{Le Sur les poètes d'Aristote}

Tout d'abord, de même que la Poétique, le Sur les poètes traitait les matières suivantes :

a. les rapports entre imitation et poésie (Arist., De poet. frg. Janko 3-4)

b. les différences entre les espèces imitatives

b.1. le moyen : visuel ou sonore ; rythme, mélodie ou discours (Arist.,

De poet. frg. Janko 6a-7, 18a-b, 20 ; cf. 21-30, 53)

b.2. l'objet : agent supérieur ou inférieur (Arist., De poet. frg. Janko 5-6b, $8-15$, cf. 53.4)

b.3. le mode : énonciatif ou dramatique (Arist., De poet. frg. Janko 16-17b, 19)

c. l'avènement et le développement des espèces poétiques

c.1. l'histoire de la comédie (Arist., De poet. frg. Janko 32-35) : la comédie a commencé en Sicile, avec Épicharme et Phormos, mais s'est développée ensuite à Athènes (Arist., De poet. frg. Janko 34 ; cf. 35)

c.2. l'histoire de la tragédie (Arist., De poet. frg. Janko 37a-42) : Théspis a introduit un acteur, Éschyle le deuxième, Sophocle le troisième (Arist., De poet. frg. Janko 38, 41-42).

Ainsi, le Sur les poètes montrait, en particulier, que l'imitation est sous-jacente à la poétique, qu'elle a un rapport à tous les poètes, et que le poète est l'imitateur d'une action complète ${ }^{23}$. Il montrait aussi que le poétique est reconnu grâce au dessein du discours, qui est l'imitation, mais non grâce au moyen, qui est le mètre. Donc, d'un côté, les dialogues socratiques et les mimes de Sophron, bien qu'ils ne soient pas en vers, sont des imitations et, de ce fait, sont appelés poèmes, et leurs auteurs, poètes (Arist., De poet. frg. Janko 43a-44e) ${ }^{24}$; de l'autre, Empédocle est comparé à Homère parce qu'il est effrayant dans son expression, parce qu'il est métaphorique, parce qu'il fait usage d'autres ressources propres à la poétique (Arist., De poet. frg. Janko 73), mais non parce qu'il imite, de sorte qu'Homère est poète, mais Empédocle, naturaliste. La mention des 
dialogues socratiques, en particulier, évoque Platon. Car, selon le Sur les poètes, Platon, qui dans sa République fait blâmer à Socrate les imitateurs et, en particulier, les poètes, a composé lui-même une espèce de discours qui est à mi-chemin entre poésie et prose, et a été doublement imitateur : d'abord, parce qu'il a imité ceux qui interrogent, ceux qui répondent, ceux qui racontent; puis, parce qu'il a imité Sophron, pour ce qui est du dramatique des dialogues, et Alexaménos de Téôs, qui a découvert l'écriture imitative 25. Ainsi, la mention des dialogues socratiques est non seulement polémique, mais c'est une provocation à laquelle les sectateurs de Platon ont été très sensibles ; Proclus, par exemple, explique pourquoi Aristote se dispute avec Platon à propos de poésie dans le Sur les poètes ${ }^{26}$, et un anonyme nous met en garde contre la jalousie d'Aristote ${ }^{27}$...

Du reste, de même que la Poétique, le Sur les poètes distinguait les espèces poétiques qui imitent un objet supérieur, à savoir l'épopée et la tragédie, par le mode d'imitation. Pourtant, tandis que la Poétique distingue l'épopée par le mode narratif et la tragédie par le mode dramatique, le Sur les poètes affirmait que la tragédie est dramatique dans toutes les parties du dialogue, sauf dans le prologue et dans les parties du messager, où elle est narrative ${ }^{28}$. Et le Sur les poètes concluait, à la vérité, que tout ce qui appartient à l'épopée appartient aussi à la tragédie, mais non l'inverse, de sorte que la tragédie est supérieure à l'épopée, conclusion ultime de la Poétique, comme on sait ${ }^{29}$.

\section{Les kritikoí et d'autres}

Or, après Aristote, la discussion sur la reconnaissance du poétique se poursuit chez les Grecs. Parmi ceux-ci, on peut signaler Héraclide du Pont (IV s. av. J.-C.), Théodecte de Phasélis (IV s. av. J.-C.), Androménide (III $\mathrm{e}$. av.J.-C.), Héracléodore (III ${ }^{\mathrm{e}} \mathrm{s}$. av. J.-C.), Pausimaque de Milet (III ${ }^{\mathrm{e}}-\mathrm{II}^{\mathrm{e}} \mathrm{s}$. av. J.-C.), Cratès de Mallos (II $\mathrm{e}$ s. av. J.-C.), Démétrios de Lacédémone ( $\left(\mathrm{II}^{\mathrm{e}}-\mathrm{I}^{\mathrm{er}} \mathrm{s}\right.$. av. J.-C.), Philodème de Gadara ( $\mathrm{II}^{\mathrm{e}}-\mathrm{I}^{\mathrm{er}} \mathrm{s}$. av. J.-C.), à côté d'autres, anonymes, dont les opinions ont été transmises par ceux-ci. Tous examinent ce qui est spécifique (ídios, idiótatos, idiótes) au discours poétique et ce qui est commun (koinós) à lui et à d'autres types de discours, afin d'établir le critère (kritérion) qui leur permette de procéder au jugement (krísis, krínein) de ce qui est poétique et de ce qui ne l'est pas ; c'est pourquoi, d'ailleurs, quelques anonymes ont été appelés kritikoí, c'est-à-dire juges de poésie (Philod., Po. I 198, 21 ; III frg. 15, 12 ; V 21, 16 ; 27, 7 ; Trac. tert. frg. f, 1, 22 ; 18, 8). Au-delà d'une recherche paisible, où chacun examine l'affaire de son point de vue, il $\mathrm{y}$ a pourtant un débat enflammé, où chacun passe en revue les opinions des autres, en les infirmant et en confirmant les siennes. Or, c'est dans ce contexte que se produit la réception du Sur les poètes, de sorte qu'il faut tenir compte de ce que les auteurs grecs jugent de la spécificité du poétique, pour pouvoir comprendre ce qu'ils jugent de l'opinion d'Aristote sur l'affaire.

Voilà comment on peut résumer quelques opinions de Démètrios, Philodème et d'autres :

a. ce qui est spécifique à la poésie est l'élocution (léxis), ou l'apparat (kataskeué, akatáskeuos) :

- un anonyme affirme que le plus spécifique et le plus important est l'élocution (Philod., Po. III frg. 54, 15-18) ;

b. ce qui est spécifique est, non pas toute l'élocution, mais une partie de celle-ci, soit le choix des mots (onómata, rhémata, léxeis), c'est-à-dire le vocabulaire (onomasía), le langage (diálektos), soit l'agencement des mots (sýnthesis) : 
- Androménide dit que ce qui est propre au poète, c'est de s'appliquer au langage et au vocabulaire (Androm., frg. Janko 17);

- un anonyme soutient que tous les poèmes, ou la plupart d'entre eux, sont composés avec des mots spécifiques; Philodème assure pourtant que ni tous les poèmes, ni même la plupart d'entre eux, ne sont composés avec des mots spécifiques, par exemple, quelques passages d'Euripide sont composés avec des mots qui ne diffèrent guère des mots communs (Philod., Po. III 10);

- un anonyme affirme que l'agencement des mots est la spécificité et l'excellence de la poésie (Philod., Po. III frg. 16, 16-17) ;

c. la pensée (diánoia, dianóema, noûs, nóema, nooúmenon) pourtant, c'est-à-dire l'affaire (prágmata), la fable (mûthos), le sujet (hypóthesis), n'est pas spécifique :

- Pausimaque dit qu'il ne faut pas approuver le poète si la fable et le sujet sont beaux (Philod., Po. I 42, 5-8), car la pensée n'appartient pas aux spécificités du poème (Philod., Po. I 43, 16-17);

d. ainsi ce qui est spécifique à la poésie est non ce que les mots signifient, mais la façon dont ils sonnent ; alors :

d.1. en général, l'élocution ne dépend pas de la pensée :

- un anonyme soutient que, si un poète introduit les mots et l'apparat qui conviennent à l'élocution, cela suffit pour dire qu'il s'agit d'un bon poète, même s'il ignore complètement la pensée ; car il n'y a aucun inconvénient si le poète ne réussit pas dans ce qui ne lui est pas spécifique. Philodème demande toutefois comment, s'il s'agit d'un poème, on le tiendra pour des mots qui signifient une pensée, plutôt que pour un air de musique (Philod., Trac. tert. frg. c 1-2);

- Androménide avance que la pensée a besoin de l'apparat pour que le poème puisse séduire (Androm., frg. Janko 3), et que le devoir du poète est non de dire ce que personne n'a dit, mais de l'exprimer d'une façon dont ceux qui ne sont pas des poètes ne l'ont jamais exprimé (Androm., frg. Janko 18);

- Héracléodore prétend que ce qui est spécifique au poète est de choisir les mots convenables et de les agencer en vue de l'explication de l'affaire (Philod., Trac. tert. 12, 19-24; cf. 13, 21-27);

d.2. en particulier, ni le choix des mots, ni leur agencement ne dépendent de la pensée : d.2.1. quant au choix des mots :

- Androménide dit qu'Homère a perfectionné la poésie grâce au choix des mots les plus beaux (Androm., frg. Janko 23 ; cf. $31 ; 33 ; 35$ ). Philodème, pourtant, objecte qu'il n'y a pas de poésie qui soit lumineuse grâce à des mots lumineux (Philod., Po. I 185, 20 - 186, 8) ; car il admet que des mots tels que khrysós, « or », et árgyros, « argent », qui signifient quelque chose de lumineux, peuvent avoir un son moins lumineux que d'autres, qui ne signifient rien de lumineux, mais il insiste sur le fait que les mots sont lumineux, non par la façon dont ils sonnent, mais par ce qu'ils signifient (Philod., Po. I 23, 7 - 24, 27) ;

d.2.2. quant à l'agencement des mots :

- un anonyme dit que le poète envisage ce qui est spécifique dans l'agencement des mots, tandis que, dans la pensée et dans les mots eux-mêmes, il ne regarde que ce qui est commun (Philod., Trac. tert. 16, 17-21);

- quelques-uns soutiennent que l'agencement des mots est bien même si le poète ignore la pensée ; d'autres, même si la pensée est entièrement absente ou désordonnée (Philod., De poem. V 22, 33 - 23, 21 ; cf. 18, 15-29);

- Pausimaque explique que, si l'agencement des mots n'est pas beau, il n'y a pas de pensée, en dépit de toute sa beauté, qui puisse assurer la qualité du poème, ou que, si 
l'agencement des mots est beau, il n'y a pas de pensée, en dépit de toute sa laideur, qui puisse nuire à la qualité du poème (Philod., Po. I 57 ; cf. 58). Héracléodore, quant à lui, dit que, si la pensée et l'élocution sont communs mais le poème est spécifique, c'est grâce à l'agencement des mots, qu'il soit bon ou mauvais (Heracleod., frg. Janko 30-31) ; - Héracléodore dit encore qu'il n'y a rien d'important si le fond est incompréhensible, ou si les mots ne sont pas les meilleurs, ou encore si le poème est obscur (Heracleod., frg. Janko $18-22$; 27), car ce qui le rend noble ou mauvais c'est l'agencement des mots (Heracleod., frg. Janko 23-24);

- Héracléodore et d'autres appellent poète celui qui agence les mots avec précision. Philodème admet que l'agencement séduit, et qu'il rentre dans le poème, mais, une fois noté que la précision ne regarde que le son, et non le sens, il objecte que, s'il n'y a que l'agencement, il faudrait appeler poème un air de musique (Philod., Po. I 199-201);

d.3. ainsi l'élocution vaut par son harmonie (harmonía), ses sons (phonaí, êkhoi), ses tons (tónoi, phthóggoi), ou encore par l'euphonie (euphonía) qui s'ensuit :

- Androménide dit que le devoir du poète est de donner un apparat aux sons et à l'harmonie (Androm., frg. Janko 19), de choisir les mots les plus beaux et de s'écarter des plus laids (Androm., frg. Janko 21);

- Héracléodore affirme que l'agencement des mots est le propre du poète, et que l'excellence du poème réside dans l'euphonie (Heracleod., frg. Janko $35 ; 37$; 41) ; ou encore, que c'est d'après l'euphonie qui naît de l'agencement que le poème devient beau ou mauvais (Heracleod., frg. Janko $32-33$; 36) ; de sorte qu'Héracléodore et d'autres dirigent leur attention, non vers l'agencement, mais vers la sonorité qui en résulte (Philod., Po. V 24, 27-31);

- l'agencement des mots et l'euphonie qui s'ensuit doivent être travaillés comme la seule chose spécifique, et la pensée et les mots rester en dehors de l'art et donc être chose commune : c'est ce qui est écrit chez tous les kritikoí comme sur la pierre (Philod., Trac. tert. 17, 1-9 ; cf. frg. b 1, 1 ; Po. I 132, 27 - 133, 3 ; V 21, 15-16 : taîs [...] euphoníais tôn kritikôn).

Alors, ceux qui ont comme critère le sens des mots concluent que le jugement de ce qui est poétique s'effectue grâce à la pensée; en revanche, ceux qui adoptent comme critère la sonorité des mots concluent que ce jugement s'opère grâce à la sensation (aisthesis) ou la perception (kataleptós, katalambánein) de l'ouïe (akoé). Ce sont ces deux critères qu'Androménide distingue, quand il dit que la sensation sympathétique qui se produit par la pensée diffère de celle qui se produit par l'ouïe (Androm., frg. Janko 2; cf. Demetr. Lac. I 3, 3 : akoês). Ce sont ces deux jugements qu'un anonyme distingue, quand il affirme que le jugement sur la qualité du poème dépend de la pensée, mais que d'autres disent que le jugement se produit d'après l'ouïe, de sorte que ce qui guide et importe est le plaisir de l'ouïe (Philod., De Po. III frg.54, 6-15). Quand pourtant Androménide dit que la pensée est juge avec l'ouie, Démétrios demande s'il y a des choses dans le poème dont l'ouie juge, et d'autres dont la pensée juge (Androm., frg. Janko 6 ; Demetr. Lac. I 14, 3 - 15, 8).

Ainsi ceux qui supposent qu'on juge de ce qui est poétique par la pensée considèrent le critère de jugement comme dépendant de la raison (lógos); en revanche, ceux qui supposent qu'on en juge par l'ouïe considèrent ce critère comme irrationnel (álogos). Héracléodore, par exemple, dit que l'ouïe est séduite par les poèmes, et que le plaisir des poèmes appartient par nature à l'agencement des mots (Heracleod., frg. Janko 7-8). Ainsi, les kritikoí disent qu'on considère les mètres et qu'on en juge par l'expérience, de 
sorte que l'ouïe témoigne des mètres, et ne s'intéresse guère à la pensée, qui est étrangère à l'art ; ou encore, ils disent que les mètres ne peuvent pas être reconnus par la raison, mais par une ouïe adroite. Il y en a pourtant qui ne sont pas d'accord avec ceux-là. Cratès, par exemple, dit qu'on juge du poème, non quand il plaît à l'ouïe, mais quand il est façonné d'après la règle rationnelle de l'art (Philod., Po. V 27, 3-13; cf. 27, $18-21 ; 27,35-28,4)$. À la vérité, selon Cratès, on ne doit juger ni de ce qui s'adresse à la sensation, ni de la pensée du poème, mais des aspects rationnels du poème par la sensation, et on doit juger par la pensée, mais non de la pensée elle-même (Philod., Po. V 28, 20-29; cf. Trac. tert. frg. a 1, 1-7). Démétrios, quant à lui, accuse Androménide de faire violence à l'argument, quand celui-ci dit qu'on juge du poème aussi bien par la partie irrationnelle de la pensée que par l'agencement des mots et la mémoire (Androm., frg. Janko 5 ; Demetr. Lac. I 13, 1-8). Philodème enfin avertit de ce que, si on agence les mots pour l'ouïe, on ne les agence pas d'après la connaissance (Philod., Po. III 9, 18-19). Ainsi, quand un anonyme affirme que et l'ouïe et la pensée jugent de ce qui est mélodieux, Philodème soutient qu'il se trompe sur ce qu'il dit des sons, s'il s'adresse à d'autres qui s'intéressent aux aspects rationnels de la poétique (Philod., Po. III frg. 16). Ainsi, quand quelques-uns disent que l'agencement des mots, ou plutôt l'euphonie qui en résulte est perçue, non par la raison, mais par la dextérité de l'ouïe, qui est irrationnelle, Philodème ne prend pas au sérieux leur opinion (Philod., Po. V 23, 21 - 24, 23).

\section{Le Sur les poètes selon les kritikoí et d'autres} Sur les poètes; en particulier, c'est à la lumière de la discussion sur le critère de reconnaissance du poétique qu'ont été revues les opinions d'Aristote sur le moyen et le mode de l'imitation poétique. C'est cette dispute, enfin, que la leçon de Diomède sur les genres et espèces de poèmes semble renfermer.

S'agissant du moyen de l'imitation poétique, Aristote dit que ce qui la distingue des autres imitations, c'est le discours simple, soit en vers, soit en prose, de sorte que, pour qu'il soit poétique, le discours doit être nécessairement imitatif, et il peut être par accident en vers; puis il illustre le discours imitatif par les mimes de Sophron, composés en prose, et le discours non imitatif par l'histoire naturelle et l'histoire, c'està-dire par Empédocle, qui pourtant a composé en vers, et par Hérodote, même si son ouvrage est transposé en vers. Or, que ces opinions aient été traitées par Philodème, Démétrios et d'autres, c'est ce qui ressort de plusieurs passages de ces auteurs. Quant à la distinction entre le poétique et le mètre, par exemple, un anonyme avertit de ce qu'une chose est le poietés, et une autre l'emmetropoiós (Philod., Po. I 46, 1-3). Des mimes de Sophron, Héracléodore dit qu'ils sont parfois, de même que les compositions d'autres mimographes, appelés poèmes, et non mimes, et leurs auteurs, poètes de mimes (Heracleod., frg. Janko 11-12) ; Démétrios, quant à lui, cite quelques anonymes qui traitent les mimes de Sophron (Demetr. Lac. II 55-60); Philodème enfin dit que, contrairement à la tragédie, qui est composée en vers, le mime est composé en prose (Philod., De poem. IV 108, 8-10). Dans un passage très fragmentaire du Sur les poèmes de Philodème (I 53), on peut déceler une distinction entre poésie (cf. I 53, 14 : poetikôn ; 53, 16: póe [ma) et histoire (cf. I 53, 21 : histor[ías), ou entre histoire et tragédie (cf. 53, 20 : tragikén), établie d'après le critère de l'imitation (cf. 53, 26: míme[si]n) ou de l'utilité (cf. I 53, 21-22: khresímas). Enfin, sur la transposition de la prose en vers, Philodème

Interférences, 6 | 2012 
imagine quelqu'un qui raconte en prose une fable qui avait été racontée en vers (Philod., Po. I 203, 15-17).

Cependant, ces opinions d'Aristote sont revues par Philodème, Démétrios et d'autres, à la lumière de la distinction entre critère rationnel et critère irrationnel. Ainsi, parce qu'Aristote dit que le moyen de l'imitation poétique est le discours simple, soit en vers, soit en prose, ils jugent qu'il écarte de l'imitation l'aspect acoustique du mètre, et la restreint à l'aspect sémantique du discours (lógos) qui, comme Aristote lui-même le définit, est un son vocal signifiant ${ }^{30}$, ils concluent alors qu'Aristote adopte un critère rationnel de reconnaissance du poétique. C'est pourquoi les kritikoi et d'autres, quand ils établissent le critère de jugement du poétique sur l'aspect sonore de l'élocution, et non sur l'aspect signifiant du discours, prennent une position qui va à l'encontre de la position aristotélicienne. Ainsi, d'un côté, le discours, sur lequel s'établit le critère d'Aristote, parce qu'il est signifiant, peut ressembler à l'action des agents et donc être proprement imitatif; de l'autre, l'euphonie, sur laquelle s'établit le critère des kritikoí, parce qu'elle est acoustique, peut ressembler aux sons des choses et donc être proprement onomatopéïque. Quoi qu'il en soit, Philodème prétend réfuter les deux positions, puisqu'il se méfie complètement du pouvoir imitatif du poème ${ }^{31}$. Ainsi, quand un anonyme dit que le poète suit les mètres, mais qu'il le fait d'après la pensée, Philodème objecte que le poème est irrationnel (Philod., Po. III 4 ; cf. 12, 25) ; autrement dit, Philodème prend les mètres du point de vue de l'euphonie, de façon à objecter que le poème est perçu par l'ouïe, mais non par la pensée. Pourtant, parce que le vers est irrationnel, Philodème dit qu'il ne peut être imitatif (Philod., Po. III 12, 24-26; cf. 4, 27); en d'autres termes, parce que le mètre est un aspect acoustique, et non sémantique, il peut simuler les sons, mais non les actions. Ainsi, Philodème, d'un côté, trouve insensé de chercher la ressemblance de l'élocution avec l'affaire auquel elle se réfère; de l'autre, il trouve absurde d'accorder au poétique la connaissance des discours qui conviennent à chaque personnage (Philod., Po.V 34, 33-35, 32). C'est pourquoi Philodème dit que la spécificité du poétique n'est ni toute l'imitation, ni l'imitation d'une action (Philod., Po. IV 106-107), c'est-à-dire ni l'imitation acoustique des sons, ni l'imitation sémantique des actions.

Quand ils examinent la différence entre poésie et prose (pezòs lógos, logoeidés, pezográphos), Philodème, Démétrios et d'autres concluent la même chose qu'Aristote, à savoir qu'elles ne s'opposent pas nécessairement l'une à l'autre. Les raisons avancées sont pourtant très diverses. Car Aristote conclut que le poétique ne se distingue pas de la prose parce qu'il croit que l'imitation se produit par la signification du discours, et non par la sonorité du mètre; Philodème et d'autres concluent de même, mais parce qu'ils croient que l'euphonie est commune à la poésie et à la prose. Ainsi, quand un anonyme dit que l'élocution fuit le prosaïque, Démétrios admet qu'on emploie des mots barbares et désordonnés dans les poèmes, mais il revendique qu'on emploie des mots qui échappent à l'usage commun, par exemple des mots composés, dans la prose (Demetr. Lac. II 14, 5-11; 18, 1-6). Ainsi, quand un anonyme dit que concision et brièveté, vraisemblance et clarté sont propres à la poétique et au poète, Philodème objecte que les prosateurs aussi recherchent concision et clarté, de sorte que ces qualités ne sont pas spécifiques à la poétique ni au poète (Philod., Po. V 6, 12-7, 20 ; cf. 30, 6-25; 31, 7-32). Théodecte évoque des anciens rhéteurs, pour lesquels le sujet est commun à la prose et aux poèmes, dont la spécificité est le vocabulaire (Philod., Po. III frg. 15, 15-31). La diversité des raisons invoquées se manifeste aussi dans les exemples par lesquels chacun illustre le poétique. Car Aristote exclut Hérodote du nombre des 
poètes, non parce qu'il compose en prose, mais parce qu'il n'est pas imitateur; Héracléodore pourtant appelle poème l'ouvrage d'Hérodote, parce que, bien qu'il soit composé en prose, il affiche un agencement de mots euphonique (Heracleod., frg. Janko 10).

Quant au mode de l'imitation poétique, Aristote dit que, dans la tragédie, le narratif se retrouve dans les parties des messagers, et le dramatique dans les autres; Philodème pourtant remarque qu'alors la distinction entre le narratif et le dramatique ne sert pas à distinguer la tragédie de l'épopée (Philod., Po. IV 112, 3-113, 14). Bien plus, Philodème évoque un anonyme qui recommande au poète une élocution qui convienne aux personnages; puis il objecte que ceci est commun aux discours en prose, du moins à l'histoire et au dialogue (Philod., Po. V 34, 33 -35, 6). En bref, selon Philodème, la distinction entre mode narratif et mode dramatique d'imitation ne suffit pas à distinguer les espèces poétiques les unes des autres (épopée et tragédie), ni celles-ci des espèces en prose (histoire et dialogue).

De même que le problème de la distinction entre poésie et prose, celui de la distinction entre les espèces poétiques est examiné, non du point de vue de l'imitation, mais du point de vue de l'élocution, c'est-à-dire d'après le choix des mots, l'agencement des mots, le mètre. Androménide dit que le caractère de l'épopée est en accord avec le vocabulaire, de même qu'il l'est avec la fable (Androm., frg. Janko 28). Héracléodore, quant à lui, dit que ni la pensée ni le langage ne suffisent à reconnaître les espèces génériques, parce qu'il n'y a pas de pensée ni de langage proprement comiques, tragiques, ou lyriques (Heracleod., frg. Janko 1-6). Ou alors, il dit que l'art poétique ne se divise pas d'après les mètres (Heracleod., frg. Janko 17). Pausimaque enfin cite quelques auteurs pour lesquels ce qui est spécifique à chaque genre poétique se manifeste par l'agencement des mots ; ensuite, pourtant, il objecte qu'il n'y a ni sujet ni langage qui soient spécifiques par eux-mêmes (Philod., Po. I 61); car le dessein de chaque genre, qu'il s'agisse de tragédie, d'iambe, de lyrique, est toujours le même, à savoir l'élocution (Philod., Po. I 77 ; cf. 83).

\section{Conclusion}

Comme la dispute d'Aristote avec Platon, celle des kritikoi et d'autres avec Aristote peut se reconstruire à partir de la leçon de Diomède sur les genres et espèces de poèmes. Tout d'abord, Diomède ouvre le Livre III de l'Ars grammatica, auquel appartient la section intitulée De poematibus, par une autre section intitulée De poetica, où il définit poétique ainsi que poème et poésie (GL I 473, 15-20). Or, c'est justement aux kritikoí et à d'autres que remonte la division de la poétique en trois eíde, "aspects». En effet, Néoptolème de Parion (III ${ }^{\mathrm{e}} \mathrm{s} . \mathrm{av}$. J.-C.) énumère trois aspects de la poietiké: poíema, poíesis et poietés (Philod., Po. V 14, 5-11); de même, Androménide dit que le poète, le poème et la poésie sont des aspects de l'art (Androm., frg. Janko 24), et Cratès en convient avec Androménide (Philod., Po. I 132, 19-27).

De même, la distinction de Diomède entre l'espèce historiké et la didaskaliké peut être comparée à la discussion des kritikoí et d'autres sur l'utilité de la poésie. Comme il a été dit, certains croient que toutes les pensées sont communes au discours poétique et à d'autres sortes de discours, et d'autres croient que quelques pensées sont spécifiques au discours poétique. Parmi ceux qui expliquent quelles sont ces pensées, certains croient qu'elles sont l'opinion commune, et d'autres qu'elles sont l'extraordinaire; ou encore, 
qu'elles sont des choses savantes ou utiles. Androménide, par exemple, dit que le savant doit examiner la vérité, et le poète ce qui est estimé de la plupart des gens (Androm., frg. Janko 12 ; cf. 10-11). Héracléodore et Cratès, quant à eux, sont d'avis que les poètes empruntent les uns aux autres non seulement le langage, mais aussi les pensées, qui peuvent être savantes, ou extraordinaires, ou impossibles (Philod., Po. I 195, 8-17; Heracleod., frg. Janko 9). Philodème enfin dit que les desseins de l'excellence poétique sont bien établis : dans l'élocution, imiter un langage qui enseigne des choses utiles, et, dans la pensée, participer à la fois à la pensée des savants et à celle de la foule (Philod., Po. V 25, $34-26,20)$. Le rapport entre poésie et enseignement, et en particulier entre poésie et enseignement de choses utiles, est polémique; car quelques-uns objectent qu'il n'est pas spécifique à la poésie, à partir du moment où il est commun à l'histoire et à la philosophie. Ainsi, dans un passage du Des poèmes de Philodème cité plus haut (I 53), on peut déceler la distinction entre poésie (cf. I 53, 14 : poetikôn; 53, 16: póe[ma) et histoire (cf. I 53, 21: histor[ías), ou entre histoire et tragédie (cf. 53, 20: tragikén), d'après le critère de l'imitation (cf. 53, $26:$ míme [si]n) ou de l'utilité (cf. I 53, 21-22: khresímas). De même, dans un passage perdu du Des poèmes, Héraclide du Pont (cf. Philod., Po. V 3, 14-16) aurait dit qu'il est propre à la poésie d'être éducative. Philodème pourtant objecte qu'alors ni les philosophes, ni les rhéteurs ne seraient éducatifs, tandis que, bien au contraire, plusieurs sortes de discours en prose sont éducatifs, par exemple les discours protreptikoí, epanetikoí, paramythetikoí, nouthetetikoí (Philod., Po. V 1-3). Ainsi la distinction entre l'espèce historiké et la didaskaliké de Diomède renferme un débat sur le dessein éducatif de la poésie, qui a été entrepris par les kritikoí et d'autres, mais qui peut concerner Aristote, qui justement distingue la poésie de l'histoire et de la philosophie.

\section{BIBLIOGRAPHIE}

\section{Textes anciens}

Aristote, La Poétique, éd. par R. Dupont-Roc et J. Lallot, Poétique 27, Paris, 1980.

Démétrius Lacon, La poesia, ed., trad. e commento a cura di C. Romeo, La scuola di Epicuro 9, Naples, 1988.

Diomedis artis grammaticae libri III, ex charisii arte grammatica excerpta, ex recensione H. Keil, Hildesheim, 2002.

Philodème, Il quinto libro della poetica: PHerc. 1425 e 1538, ed., trad. e commento a cura di C. Mangoni, La scuola di Epicuro 14, Naples, 1993.

Philodème, On Poems. Book one, ed. with introd., transl. and commentary by R. Janko, Philodemus 1, 1, Oxford, 2000.

Philodème, On Poems. Books 3-4: With the Fragments of Aristotle, On Poets, ed. with introd., transl. and commentary by R. Janko, Philodemus Translation Series 1, 3, Oxford, 2011. 
Platon, Respublica, in I. Burnet (éd.), Platonis opera. II, Tetralogias III-IV continens, Scriptorum classicorum bibliotheca Oxoniensis, Oxford, 1902, p. 327-621.

\section{Textes modernes}

JANKO R. 1984, Aristotle on Comedy: Towards a Reconstruction of "Poetics II", Londres.

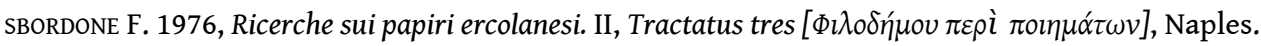

\section{NOTES}

1. Cf. Diom., GL I 482 14-17 : quod Graeci [...], quod Graeci [...] dicunt, [...] quod Graeci [...] appellant.

2. GL I 484, 1-2 : épos estìn periokhè theíon te kaì heroikôn kaì anthropínon pragmáton.

3. GL I 487, 12 : tragoidía estìn heroikês týkhes perístasis.

4. GL I 488, 4-5 : komoidía estin idiotikôn pragmáton akíndynos periokhé.

5. GL I 491, 15-16 : mîmós estin mímesis bíou tá te sygkekhoreména kaì asygkhóreta periékhon.

6. GL I 484, 1 : a Graecis ita definitum est ; 488, 4 : apud Graecos ita definita ; 491, 14-15: a Graecis ita definitus.

7. GL I 487, 11-12: a Theophrasto ita definita est ; frg. Fortenbaugh 708.

8. Ammon., In Int. 4, 17a : dià [...] éti te makrologías kaì brakhylogías; Th., frg. Fortenbaugh 78.

9. D.H., Comp. 22, 6 : hékist' antherá ; cf. 22, 8 : ánthesi.

10. D.H., Dem. 18, 7 : antheràn dè [...] ek pantòs axiôn eînai tèn diálekton; cf. Isocr. 3, 4; Th., frg. Fortenbaugh 691.

11. Arist., Po. 1, 1447 a 16 : diaphérousi dè allélon trisín ; 3, 1448 a $24:$ En trisì dè taútais diaphoraîs.

12. Arist., Po. 3, 1448 a 19-24 : kaì gàr en toîs autoîs kaì tà autà mimeîsthai éstin hotè mèn apaggéllonta è héterón ti gignómenon hósper Hómeros poieî è hos tòn autòn kaì mè metabállonta-, è pántas hos práttontas kaì energoûntas toùs mimouménous.

13. De même, dans le chapitre 22 , quand il conclut l'exposé de la tragédie, Aristote dit qu'il va parler de l'imitation dans le drame, et, dans le chapitre 23, quand il passe à l'exposé de l'épopée, il annonce qu'il va traiter de l'imitation narrative (cf. Arist., Po. 22, 1459 a 15-23, 1459 a 7 : Perì mèn oûn tragoidías kaì tês en tôi práttein miméseos [...]. Perì dè tês diegematikês kaì en métroi mimetikês). Cf. 5, 1449 b 9-12: tôi dè [...] apaggelían eînai, taútei diaphérousin ; 6, 1449 b 26 : drónton kaì ou di' apaggelías.

14. Arist., Po. 1, 1447b 13 : hoi ánthropoí.

15. Plat., $R$. II $380 \mathrm{c}$ : en métroi [...] áneu métrou.

16. Arist., Po. 9, 1451 b 1 : è émmetra [...] è ámetra.

17. Plat., R. III 393d : phráso dè áneu métrou : ou gár eimi poietikós.

18. Plat., R. II 380c : toùs légontas légein kaì toùs poioûntas poieîn; 383 a : kaì légein kaì poieîn; III $387 \mathrm{c}$ : lektéon te kaì poietéon; $390 \mathrm{a}$ : en lógoi è en poiései ; $392 \mathrm{a}:$ kaì poietaì kaì logopoiò ; $397 \mathrm{c}$ : hoi poietaì kaì hoí ti légontes (cf. $392 \mathrm{~b}$ : áidein te kaì mythologeîn; $365 \mathrm{e}$ : ék te tôn lógon kaì tôn genealogesánton poietôn; $392 \mathrm{~d}$ : hypò mythológon è poietôn ; $394 \mathrm{~b}$ : tês poiéseos te kaì mythologías).

19. T. Coislin. 1-2 : Tês poiéseos he mèn amímetos : historiké, paideutiké (= hyphegetiké, theoretiké), he dè mimetiké : tò mèn apaggeltikón, tò dè dramatikòn kaì praktikón (= komoidía, tragoidía, mímous, satýrous).

20. La première fois qu'il définit le mètre, Diomède distingue trois metra: le bioticon, propre à la vie et aux réunions, le poeticon, propre aux mots et aux vers des poètes, et le commune, propre à la règle et à la science musicale (GL I 474, 9-24; cf. 494, 3-8). Donc on peut conclure que, pour Diomède, ce ne sont pas toutes les compositions dans le mètre qui sont poétiques, mais on ne peut pas conclure que les compositions poétiques ne sont pas toutes dans le mètre. De même, de 
la distinction entre poétique, poésie et poème, on ne peut pas voir si, pour lui, le poème doit nécessairement être composition dans le mètre, ou peut par accident être composition sans mètre (GL I 473, 16-20). La définition du poète comme celui qui fabrique des vers (GL I 491, 18 : is qui versum facit dictus poietés) est incidentelle, ou plutôt, Diomède s'en sert pour montrer par induction que, de même que poietés, «celui qui fabrique", s'applique en particulier à celui qui fabrique des vers, bien que plusieurs autres artisans fabriquent quelque chose, mimus, « celui qui imite ", se réfère en particulier à celui qui imite un certain genre de vie, bien qu'il ne soit pas le seul à imiter quelque chose.

21. Qu'il s'agisse de compositions dans le mètre, en particulier, dans l'hexamètre héroïque, c'est ce qui ressort aussi bien des fragments du Catalogue des femmes d'Hésiode (Hes., Cat. frg. Evelyn-White 1-100) que des fragments du Sur la nature (Emp., De nat. frg. DK B 1-111) et des Purifications (Emp., Purif. frg. DK B 112-154) d'Empédocle.

22. GL I 299, 17-18: tertia pedum qualitatem, poematum genera mètrerumque tractatus plenissime docet; cf. 473, 4-8: in hoc vero tertio libro [...] metra [...] quibus pedum qualitatibus conpositionisve metricae observationibus regantur, certis rationibus edocebo.

23. Arist., De poet. frg. Janko 4 : mímes[i]n keîsthai [têi] poietikêi ; 3 : pe]rì [mímesi]n katà p[á]nton; 45 : [poie]tès mimetés est [i prá]xeos tele(i)as.

24. Arist., De poet. frg. Janko $44 \mathrm{a}$ : oukoûn oudè emmétrous <óntas> toùs kalouménous Sóphronos mímous mè phômen eînai lógous kaì miméseis; $44 \mathrm{~d}$ : "poéma[t" eînai lé]getai, kaì mè " $m$ [îmoi", kaì] hoi syntithén[tes autà] "mímon poe[tai]".

25. Arist., De poet. frg. Janko 43 a : kaì eiségage lógou idéan kerasámenos ek poiéseos kaì psilometrías, <mimoúmenos> toùs erotôntas kaì apokrinoménous kaì diegouménous; $43 \mathrm{~b}$ : tèn tôn lógon idéan autoûi (sc. Plátonos) metaxù poiématos eînai kaì pezoû lógou; $44 \mathrm{a}$ : oukoûn oudè emmétrous <óntas> toùs kalouménous Sóphronos mímous mè phômen eînai lógous kaì miméseis, è toùs Alexamenoû toû Teíou toùs prótous graphéntas tôn Sokratikôn dialógon; $44 \mathrm{~b}$ : heuretés [...] toû mimetikôs gráphein ; $44 \mathrm{c}:$ ka<tà> tò dramatikòn tôn dialógon [...] dramatikoùs dialógous; $\mathrm{cf} .44 \mathrm{e}$.

26. Arist., De poet. frg. Janko 56 : kaì tôi <Aristotélei> [...] kaì toîs hypèr tôn poiéseon toúton agonistaîs tôn pròs Plátona lógon.

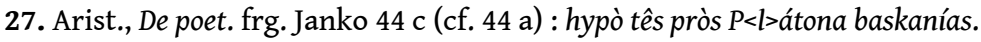

28. Arist., De poet. frg. Janko 16 : epei) [pánta prát]to[u]sin è tà pleîsth' hoi tôn trag[i]kôn hypokritaì pl[è ]n [p]ro[l]ó[goi]s; 17 a : (kaì tôi) tês mèn tragoid[i]as tó te apaggéllein en tôis aggélois kaì tò praktikòn en toîs állois (hypárkhein méresin), en d[è tô̂] épesi tò mónon [a]paggéllein; $17 \mathrm{~b}:[$ th]etéon tèn apagg[el]ían hos mórion tês drastikês.

29. Cf. Arist., De poet. frg. Janko 19 : pánta (mèn gàr) en têi tragoidíai t[à] kaì en ekeínei[i], tounantían d' oukh hypárkhein; Po. 26, 1462 a 14 : pánt' ékhei [sc. tragoidía] hósaper he epopoiía.

30. Arist., Po. 20, 1457 a 23-24 : Lógos dè phonè synthetè semantikè.

31. Philod., Po. V 2613-2615 : tò poíema d' estì tò mimoúmenon hos endékhetai ; cf. 25, 34 - 26, 20.

\section{RÉSUMÉS}

L'article analyse le De Poematibus de Diomède à travers l'examen des sources platoniciennes et aristotéliciennes du grammairien et montre que le livre se fait le relais d'une dispute philosophique sur la spécificité du poétique où Diomède, en réfléchissant sur les notions de genera et de species, fait en réalité état d'un débat sur la reconnaissance du poétique. 


\section{INDEX}

Keywords : grammarians, mimesis, poetics, rhetoric, enunciator, tragedy, comedy, etymology, narrative, prosa, definition of literary genres, persuasion, poetic awareness, metric awareness, criticism

Mots-clés : grammairiens, conscience poétique, conscience métrique, généricité, énonciateur, poétique, rhétorique, persuasion, tragédie, comédie, étymologie, narration, prose, définition des genres littéraires, critique

nomsmotscles Alexaménos de Téos, Ammonius Philosophus, Androménide, Aratos, Aristote, Chérémon, Cratès de Mallos, Démétrios de Lacédémone, Denys d'Halicarnasse, Diomède, Empédocle, Épicharme, Eschyle, Héracléodore, Héraclide du Pont, Hérodote, Hésiode, Homère, Horace, Isocrate, Lucrèce, Lysias, Pausimaque de Milet, Philodème de Gadara, Phocylide, Platon, Proclus, Sophron, Théodecte de Phasélis, Théognis, Théophraste, Thrasymaque, Thucydide, Virgile, Xénarque

\section{AUTEURS}

\section{MARCOS MARTINHO}

Université de São Paulo (Brésil) 